\title{
Carcinoid Right Heart Disease
}

\author{
Alejandro Sanchez-Nadales ${ }^{\mathrm{a}, \mathrm{d}}$, Valentina Celis-Barreto ${ }^{\mathrm{b}}$, Andrea Anampa-Guzman ${ }^{\mathrm{c}}$, \\ Mariani Salazar-Leon ${ }^{\mathrm{a}}$, Pamela Contreras-Chavez ${ }^{\mathrm{a}}$
}

\begin{abstract}
Carcinoid heart disease is an unusual cause of right heart failure. We present the case of a 53-year-old man with a history of metastatic carcinoid tumors originating from the appendix, status postchemotherapy, and liver embolizations. The patient presented with a right-sided valvular disease with severe pulmonic valve regurgitation, right ventricle dilation and pulmonary hypertension. He had carcinoid syndrome well controlled with long-acting lanreotide. $\mathrm{He}$ underwent tricuspid valve and pulmonary valve replacement with a stented bioprosthetic valve, maze and cardio-septal right ventricular outflow tract patch with an improvement of ventricular dysfunction. These findings supported the diagnosis of carcinoid heart disease presenting with pure right heart failure. The patient was symptomfree due to lanreotide, but ultimately, valve surgery is the preferred definite treatment in suitable patients. Carcinoid heart disease requires a high index of suspicion, and valve surgery is the only definitive treatment.
\end{abstract}

Keywords: Carcinoid heart disease; Right-sided heart failure; Tricuspid valve replacement; Pulmonary valve replacement; Echocardiography

\section{Introduction}

Carcinoid heart disease is a rare cardiac manifestation of advanced carcinoid tumors and carcinoid syndrome, typically involving right heart valves (tricuspid valve as the most affected) and resulting in right heart failure. The affected heart valves are thickened and retracted, resulting in valvular regurgitation, and less commonly, valvular stenosis. Left heart valve involvement is rare. Carcinoid tumors are uncommon slowgrowing neuroendocrine malignant tumors, beginning in the

Manuscript submitted March 11, 2020, accepted March 16, 2020

aDepartment of Medicine, Advocate Illinois Masonic Medical Center, Chicago, IL, USA

bDepartment of Medicine, Mount Sinai Medical Center, Miami, FL, USA

${ }^{\mathrm{c}}$ Faculty of Medicine, Universidad Nacional Mayor de San Marcos, Lima, Peru

${ }^{\mathrm{d} C}$ Corresponding Author: Alejandro Sanchez-Nadales, Department of Medicine, Advocate Illinois Masonic Medical Center, Chicago, IL, USA. Email: Alejandro.sanchez2@advocatehealth.com

doi: https://doi.org/10.14740/jmc3452 gastrointestinal tract or bronchopulmonary system. Carcinoid syndrome refers to the development of vasomotor symptoms, such as flushing, diarrhea and bronchospasm in response to secretion of vasoactive hormones by a carcinoid tumor [1-3].

Echocardiography is used to screen for cardiac involvement in all patients with a carcinoid tumor or carcinoid syndrome. Dyspnea and fatigue highly suggest some degree of cardiac involvement, with levels of pro-brain natriuretic peptide (NT-proBNP) usually elevated (NT-proBNP > $260 \mathrm{pg} / \mathrm{mL}$ or $>30 \mathrm{pmol} / \mathrm{L}$ ). The chest X-ray is typically normal, but findings may include cardiac enlargement (especially right-sided chambers) and pleural effusion. Cardiac magnetic resonance imaging (MRI) can be used if the severity of valvular regurgitation is dubious [4].

Valve replacement surgery is recommended to be performed in patients with symptomatic and severe valvular dysfunction. Medical therapy is provided to patients scheduled for valve replacement (including somatostatin analogs such as octreotide) weeks before the surgery to control tumor related symptoms and to prevent carcinoid crisis. Valve replacement increases survival in patients with carcinoid heart disease. Patients are followed up every 3 to 6 months after valvular surgery with a clinical exam, laboratory tests and echocardiography [5-8].

\section{Case Report}

A 53-year-old man with a history of myocardial infarction 6 years ago, right after a carcinoid tumor was found while undergoing work up for symptoms of chronic diarrhea and persisted wheezing. The tumor originated from the appendix, and over the years, the disease progressed to a carcinoid tumor of the liver. At that time, the patient underwent liver embolization with postoperative chemotherapy. The disease was controlled with monthly lanreotide injections, until he developed symptoms of right heart failure 1 year ago, including ascites, peripheral edema and shortness of breath. Upon further evaluation, multiple metastases were found in an abdominal computed tomography (CT) with contrast (with additional images suggestive of severely dilated right ventricle) along with a new diagnosis of liver cirrhosis (Figs. 1, 2). Cardiac work up revealed new onset atrial fibrillation in a 12-lead electrocardiogram, and a 2D echocardiogram with evidence of severely dilated right ventricle and severe tricuspid and pulmonary regurgitation (Figs. 3, 4). Due to the severity of carcinoid heart disease and profound symptoms, he was referred for evaluation to perform valvular replacement and Maze procedure. Cardiothoracic sur- 


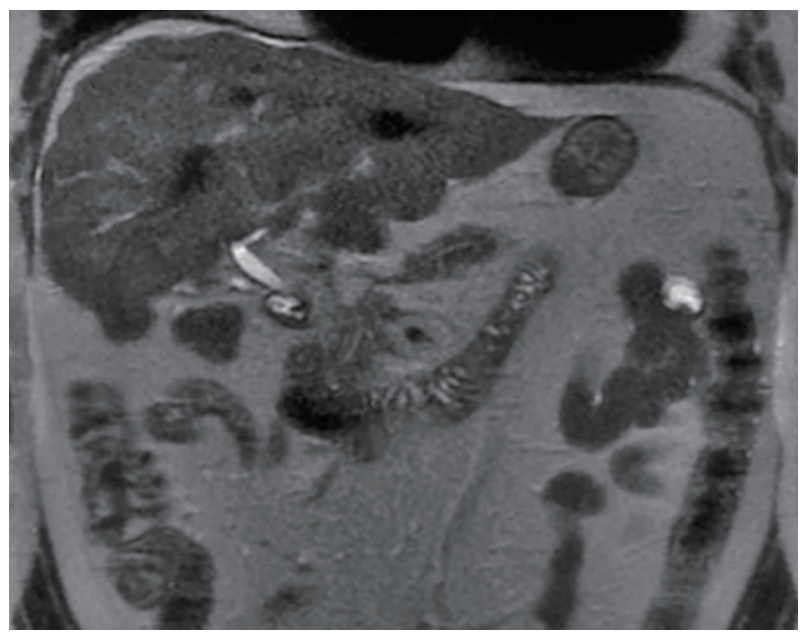

Figure 1. Cirrhotic liver morphology. Multiple low-attenuation foci in the liver (likely treated metastases). Necrotic foci in the peripancreatic space.

gery was consulted, categorizing the patient as a low-risk for open-heart surgery.

During the surgical procedure, the epicardium and the right atrium were found to be enlarged and fibrotic, and the tricuspid and pulmonic valves were sclerotic. The patient underwent a cardiopulmonary bypass with a Maze procedure, and left atrial appendix was excised. Pulmonary and tricuspid valves were replaced with a Biocor prosthesis \#27 and \#33, respectively. Finally, he was weaned from cardiopulmonary bypass. Postoperative echocardiography demonstrated wellseated and functional valves (Fig. 5). Valve remnants, both the pulmonic and tricuspid, were sent to pathology. After the procedure, the patient was started on octreotide infusion with close monitoring for the development of carcinoid crisis.

The final pathological findings included the following: pericardial fluid sac with proteinaceous material with an early organization and clusters of macrophages staining with CD68 by immunohistochemistry and mesothelialm without evidence of malignant cells; pulmonary valve with fibrosis and mild

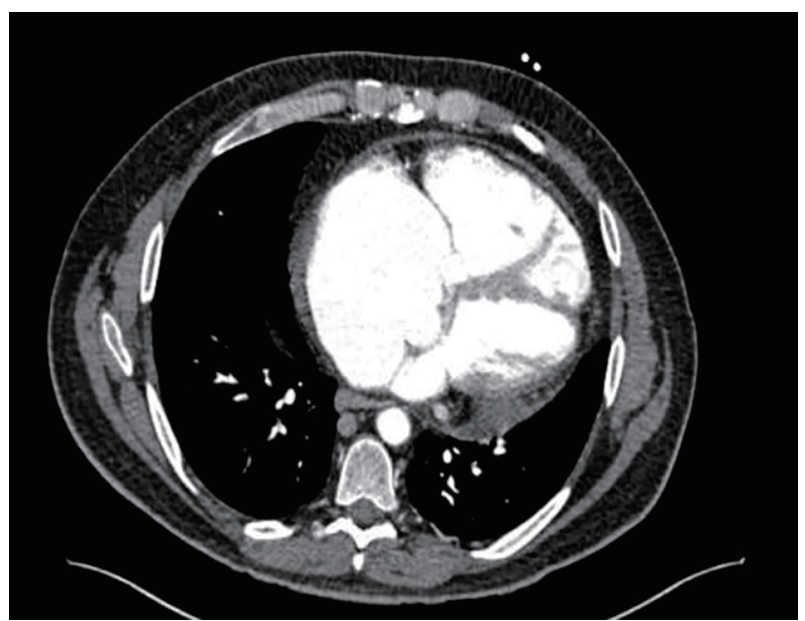

Figure 2. Severely dilated right atrium and ventricle.
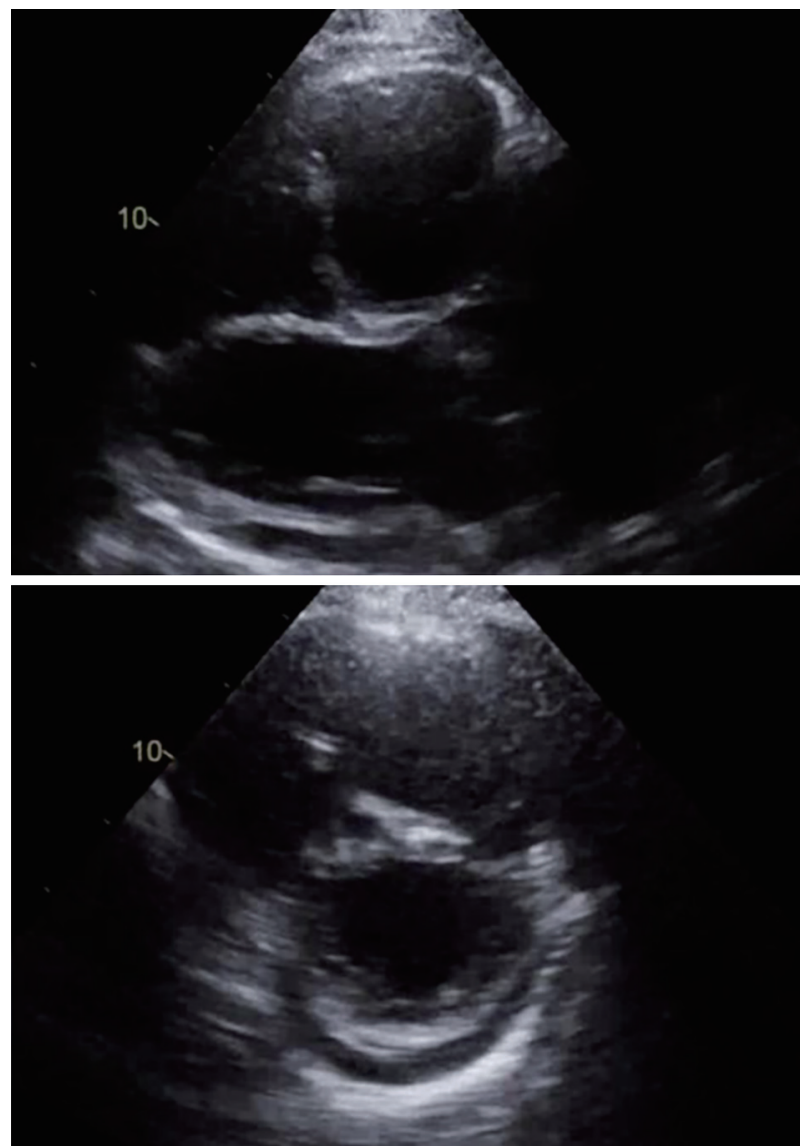

Figure 3. Right ventricle (RV) severely dilated. RV systolic tissue Doppler velocity is $9.0 \mathrm{~cm} / \mathrm{s}$. The estimated right ventricular systolic pressure is $43 \mathrm{~mm} \mathrm{Hg}$ consistent with mild pulmonary hypertension (but may be underestimated due to severe tricuspid regurgitation).

chronic inflammation (the fibrosis replaced the spongiosa layer of the semilunar valve leaflet), without evidence of giant cells or granulomata and no evidence of vegetations; left atrial appendage with findings of histologically normal myocardium.

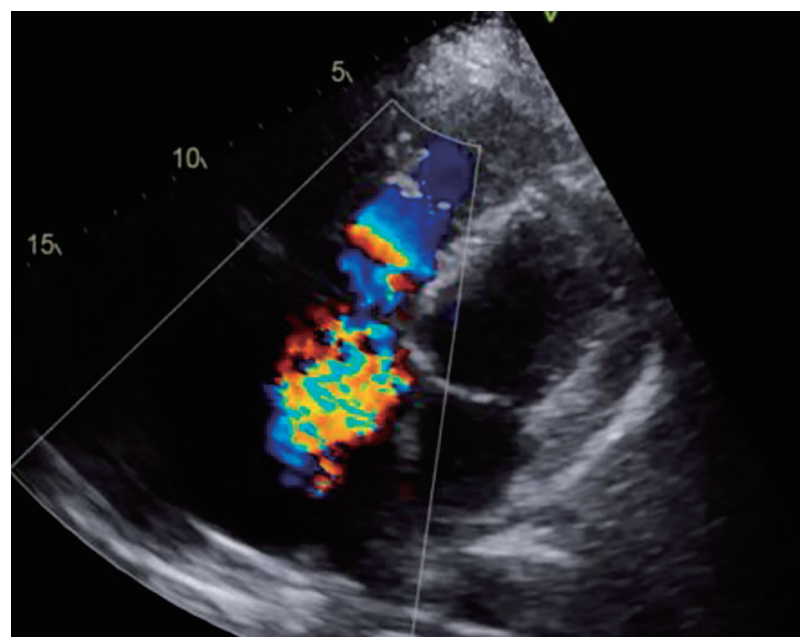

Figure 4. Severe (4+) tricuspid valve regurgitation. 


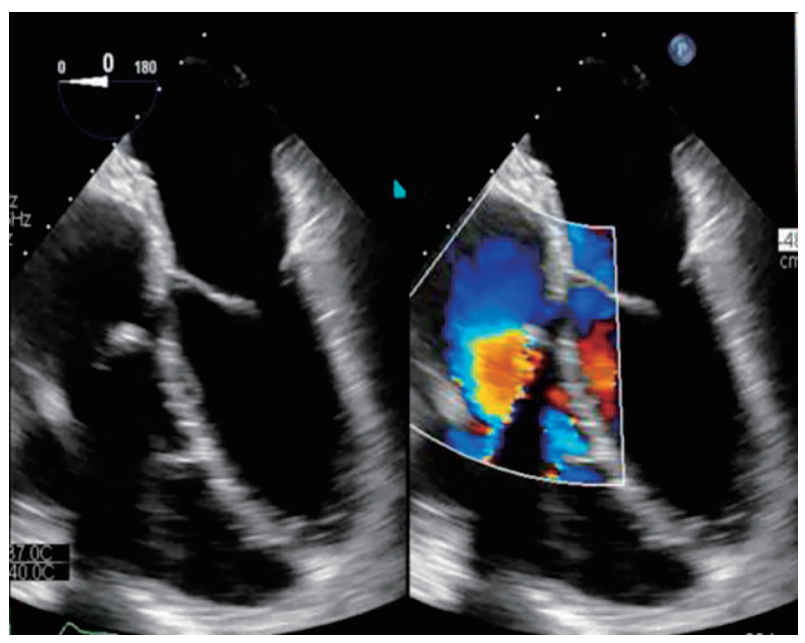

Figure 5. Status post valves replacement. Biocor prosthetic tricuspid valve size \#33. Biocor prosthetic pulmonary valve size \#27. There is no prosthetic regurgitation.

\section{Discussion}

More than half of patients with carcinoid syndrome can have carcinoid heart disease, presenting with valvular involvement and symptoms of heart failure (mostly edema, ascites and shortness of breath) [9]. Right-sided heart failure can be the initial presentation of carcinoid syndrome in up to $20 \%$ of patients. Carcinoid heart disease has characteristic plaquelike deposits of fibrous tissue, consisting of smooth muscle cells, myofibroblasts, extracellular matrix and an overlying endothelial layer. These deposits are often found in the tricuspid valve, pulmonary valve, cardiac chambers, pulmonary artery, vena cava and the coronary sinus [10]. Fibrous reactions occur most often in the endocardium of the valvular cusps; nevertheless, it can also involve the sub-valvular apparatus and the papillary muscles. This reaction leads to valve stenosis or regurgitation.

Right-sided carcinoid heart disease, like that of our patient, is the most common type of heart carcinoid disease [11]. Medical therapy is limited to symptom control and palliation. Valve replacement surgery is advised in patients with carcinoid heart disease and symptom-controlled carcinoid disease. Having surgery early in the onset of cardiac symptoms is preferable, as a delay can result in worsening of right-sided heart failure and increase the mortality risk during surgical valve replacement. Patients with progressive fatigue, worsening exercise capacity and declining right ventricle function, like in our patient, in addition to well-controlled metastatic disease, should be rapidly evaluated for valve surgery [12]. Tricuspid valve replacement is the gold standard, without the need for long-term anticoagulation when bioprosthetic valves are chosen, but these valves are highly susceptible to premature degeneration. Bioprosthetic valve dysfunction may occur from thrombosis, carcinoid plaque deposition, or accelerated degeneration [13]. Pulmonary valve replacement can also be performed due to the reduced risk of adverse right heart dilation after surgery. While most patients require replacement for right-sided valve involvement, concomitant left-sided carcinoid heart disease can sometimes lead to triple or even quadruple valve surgery [14]. Patients with carcinoid heart disease often have advanced disease at the time of the diagnosis, leading to high overall mortality and poor long-term survival [9]. Valve surgery for carcinoid heart disease should be considered for symptomatic patients with controlled metastatic carcinoid syndrome. A multidisciplinary approach is needed to achieve optimal management.

\section{Acknowledgments}

We would like to express our sincere thanks to Valeria CelisBarreto, MD and Mr. Breandan Richard Quinn for their help and assistance in the editing and proofreading of this article.

\section{Financial Disclosure}

Authors did not receive external funding.

\section{Conflict of Interest}

The authors declare that they have no competing interests.

\section{Informed Consent}

It was determined that informed consent was not necessary because data were obtained from medical records and anonymized.

\section{Author Contributions}

All authors contributed to manuscript writing and approved the final version. ASN, VCB, AAG, MSL and PCC contributed to conception and design and data analysis and interpretation. ASN contributed to the collection and assembly of data and design and data analysis and interpretation. ASN, VCB, AAG, MSL and PCC helped with data analysis and interpretation. All authors read and approved the final manuscript.

\section{Data Availability}

The data supporting the findings of this study are available from the corresponding author upon reasonable request.

\section{References}

1. Luis SA, Pellikka PA. Carcinoid heart disease: diagnosis and management. Best Pract Res Clin Endocrinol Metab. 2016;30(1):149-158.

2. Patel C, Mathur M, Escarcega RO, Bove AA. Carcinoid 
heart disease: current understanding and future directions. Am Heart J. 2014;167(6):789-795.

3. Grozinsky-Glasberg S, Grossman AB, Gross DJ. Carcinoid heart disease: from pathophysiology to treatment - 'something in the way it moves'. Neuroendocrinology. 2015;101(4):263-273.

4. Robertson RG, Geiger WJ, Davis NB. Carcinoid tumors. Am Fam Physician. 2006;74(3):429-434.

5. Lamarca A, Barriuso J, McNamara MG, Hubner RA, Valle JW. Telotristat ethyl: a new option for the management of carcinoid syndrome. Expert Opin Pharmacother. 2016;17(18):2487-2498.

6. Lafaras CT, Mandala EM, Platogiannis DN, Saratzis AN, Barbetakis NG, Paraskevopoulos PP, Ilonidis GC, et al. Evaluation of treatment with bosentan in patients with carcinoid heart disease: single center study. Onkologie. 2010;33(6):300-304.

7. Moller JE, Pellikka PA, Bernheim AM, Schaff HV, Rubin J, Connolly HM. Prognosis of carcinoid heart disease: analysis of 200 cases over two decades. Circulation. 2005;112(21):3320-3327.

8. Arnold R, Chen YJ, Costa F, Falconi M, Gross D, Grossman AB, Hyrdel R, et al. ENETS consensus guidelines for the standards of care in neuroendocrine tumors: follow-up and documentation. Neuroendocrinology. 2009;90(2):227-233.

9. Hassan SA, Banchs J, Iliescu C, Dasari A, LopezMattei J, Yusuf SW. Carcinoid heart disease. Heart. 2017;103(19):1488-1495.

10. Modlin IM, Sandor A. An analysis of 8305 cases of carcinoid tumors. Cancer. 1997;79(4):813-829.

11. Yao JC, Hassan M, Phan A, Dagohoy C, Leary C, Mares JE, Abdalla EK, et al. One hundred years after "carcinoid": epidemiology of and prognostic factors for neuroendocrine tumors in 35,825 cases in the United States. J Clin Oncol. 2008;26(18):3063-3072.

12. Connolly HM, Schaff HV, Abel MD, Rubin J, Askew JW, Li Z, Inda JJ, et al. Early and late outcomes of surgical treatment in carcinoid heart disease. J Am Coll Cardiol. 2015;66(20):2189-2196.

13. Pislaru SV, Hussain I, Pellikka PA, Maleszewski JJ, Hanna RD, Schaff HV, Connolly HM. Misconceptions, diagnostic challenges and treatment opportunities in bioprosthetic valve thrombosis: lessons from a case series. Eur J Cardiothorac Surg. 2015;47(4):725-732.

14. Bhattacharyya S, Raja SG, Toumpanakis C, Caplin ME, Dreyfus GD, Davar J. Eur J Cardiothorac Surg. 2011;40(1):168-172. 